\title{
A novel predictive model for capturing threats for facilitating effective social distancing in COVID-19
}

\author{
Salma Firdose ${ }^{1}$, Surendran Swapna Kumar², Ravinda Gayan Narendra Meegama ${ }^{3}$ \\ ${ }^{1}$ Department of Computer Science, Christ Academy Institute of Advanced Studies Christ Nagar, Bengaluru, India \\ ${ }^{2}$ Department of Electronics and Communication Engineering, Vidya Academy of Science and Technology, Kerala, India \\ ${ }^{3}$ Department of Computer Science, Faculty of Applied Sciences, University of Sri Jayewardenepura, Nugegoda, Sri Lanka
}

\begin{tabular}{l} 
Article Info \\
\hline Article history: \\
Received Mar 10, \\
Revised Jul 14, 202 \\
Accepted Aug 2, 2 \\
\hline Keywords: \\
COVID-19 \\
Deep learning \\
Machine learning \\
Pandemic \\
Social distancing
\end{tabular}

Article Info

Article history:

Received Mar 10, 2021

Revised Jul 14, 2021

Accepted Aug 2, 2021

Keywords:

COVID-19

Deep learning

Pandemic

\begin{abstract}
Social distancing is one of the simple and effective shields for every individual to control spreading of virus in present scenario of pandemic coronavirus disease (COVID-19). However, existing application of social distancing is a basic model and it is also characterized by various pitfalls in case of dynamic monitoring of infected individual accurately. Review of existing literature shows that there has been various dedicated research attempt towards social distancing using available technologies, however, there are further scope of improvement too. This paper has introduced a novel framework which is capable of computing the level of threat with much higher degree of accuracy using distance and duration of stay as elementary parameters. Finally, the model can successfully classify the level of threats using deep learning. The study outcome shows that proposed system offers better predictive performance in contrast to other approaches.
\end{abstract}

This is an open access article under the CC BY-SA license.

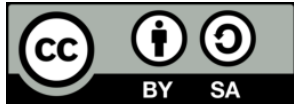

\section{Corresponding Author:}

Salma Firdose

Department of Computer Science, Christ Academy Institute of Advanced Studies Christ Nagar

Hullahalli, Begur - Koppa Road, Sakkalwara Post Bengaluru, India

Email: salmaf@caias.in

\section{INTRODUCTION}

The research study about the future transmission of the SARS-CoV-2 is a very active open research topic today [1]-[4]. A time-series data containing information's about the Human coronavirus OC43 and Human coronavirus OC43 with associated cross immunity factors are getting used for building models to predict the transmission. Studies shows that many episodes of severe pandemic wave are yet to be seen, therefore social distancing is must till 2022 [5]. Even in the event of outward purging, SARS-CoV-2 observation should be maintained because a rebirth in infection could be possible as late as 2024 . Therefore, the social distance is the mandatory requirement to be adopted for many years to come. Another dimension for human survival is country economic health, for that the activities of business need to be resumed at earliest. The full-fledged functioning of the metro and its satellite towns like Delhi, Mumbai, Chennai, and Bangalore. at earliest is a need of an hour, but balancing the act of full-fledged operation of these cities with social distancing demands some adoptable techniques which is more practical. This research proposal aims to extends the study in direction of "social distancing-oriented information sharing mechanism" on the basis of the crowdsensing technique.In the very first phase pandemic, a serious lockdown was imposed in India and even after a conditioned unlock process, emphasis was given on the social distancing, even though the trend of the infections and died people has increased. The majority of affected countries have adopted social distancing measures in the first pandemic phase, which have been worsened by the increasing trend in the number of people infected and died. The Table 1 shows the illustration of the statistics. Table 1 highlights the current statistics of the Coronavirus disease (COVID-19) on the people. 
Table 1. Number of the COVID-19 cases across India as of August 27, 2020 [6]

\begin{tabular}{llll}
\hline Deceased & Active & Recovered & Confirmed \\
\hline 61695 & 739248 & 2583063 & 3384576 \\
\hline
\end{tabular}

Therefore, irrespective of the unlocking process the social distancing is require for a prolonged period to avoid the possibility of re-infection: i) effect of social distancing: the negative trend of the GDP, loss of Jobs, changes environment of society, and scope of entertainment collectively has already impacted the socio-economic balance. The impact of prolonged social distancing will be incalculable from other ill effect on the physical, mental and economic health of the mankind [7]-[10]; ii) rationale of the solutions approach: the use of smart city technology especially mobile crowdsensing could yielded a feasible solution to make the success of the quarantine that does not impact on the decline of the economy. The technology namely mobile crowdsensing works on the data collected from the massive number sensor devices.

At present, the mechanism of the social distancing is carried out individually by using Arogya Setu mobile application. The application has certain advantages i.e: i) it can perform live detection of any user coming in contact with the infected user using Bluetooth, ii) it get updates from national healthcare authorizes with region specification. However, it has certain pitfalls too viz: i) it does not offer live tracking of the user over map although it uses global positioning system (GPS), it shows by distance and not with respect to time; ii) the outcome of infected person keeps is uncertain as it completely depends upon the report uploaded by health official, where there are also cases of negative identification; iii) it does not carry out any predictive operation; and iv) it does not offer any customized solution of social distance rather it shares similar template of information to all users. Therefore, the proposed system develops a novel framework which is meant for contributing the social distancing by the user by tracking more information details live on their handheld device. The organization of the manuscript is as follow: section 2 discusses about the existing approaches followed by discussion of identified research problem in section 3. Proposed research methodology is discussed in section 4 while system design is briefed in section 5 and result discussion in section 6. Summary of the paper is provided in section 7.

\section{RELATED WORK}

At present, there is certain attempt of research being carried out towards social distancing. The work of Mason et al. [11] has used a virtual reality technology in order to frameup a new concept of social distancing using AI. Adoption of the Wi-Fi has been reported towards alerting the individual in transportation system for enhancing social distancing concept as seen in work of Oransirikul and Takada [12]. Wiberg [13] have carried out an investigation towards both social and physical distancing. The work of Milne et al. [14] has used a stochastic model towards maintaining social distance while boarding transportation system. The work carried out by Nguyen et al. [15] have discussed about various enabling technologies towards social distancing. The work reported by Cristani et al. [16] has carried out a study towards social distancing using visual approach, which is a non-invasive analysis approach. Importance of mobile media towards social distancing is investigated by Brandtzaeg [17]. The work carried out by Pejo and Biczok [18] has implemented game theory in order to formulate better decision making in wearing mask as well as social distancing. The work carried out by Punn et al. [19] have developed a scheme for assessing social distancing using object detection and tracking concept. The work carried out by Rezaei and Azarmi [20] have developed a framework which can perform assessment of risk associated with COVID-19 using a new idea of social distancing. A closer look into various attempted approach of social distancing shows the adoption of artificial intelligence [21]-[24], machine learning [25], [26], game theory [27], [28], different variants of neural network [29], [30] when it comes to controlling the pandemic situation control.

\section{PROBLEM IDENTIFICATION}

It can be seen that there are certain dedicated attempts towards working on social distancing; however, there are certain problems associated with it viz: i) existing approaches of social distancing is basically carried out considering static set of data which is less practical; ii) adoption of various sophisticated operation in order to perform the identification of risk; iii) higher dependency of the pre-defined research environment in highly controller manner, which is less practical. Apart from this, there are some dedicated studies carried out using machine learning approach; however they have not been assessed or investigated with respect to COVID-19 case with higher threat. Hence, there is a need of a cost effective and precise computational model which offers better computational efficiency as well as they can be implemented in practical environmental scenario. The next section discusses about the solution to address the identified research problems. 


\section{PROPOSED SOLUTION}

The proposed study aims for developing a novel and simplified scheme towards controlling the pandemic situation of COVID-19 by offering a predictive outcome of the threat to the user. The proposed system is implemented as a combination of the handheld device where the framework for aggregation the contact information is carried out and cloud server where the predictive operation is carried out to let know the user about their personal level of threat. The study area chosen for this research project will city of Bangalore, where for the mobile crowd sensing the raw data generated from the accelerometer, GPS, network connectivity, camera, or microphone along with the social network traits, will be considered as these hardware sensors are embedded in mobile devices. The insights extracted from these data analysis may include the followings:

- Limitations of the freedom of movement of persons

- Opening to the public unauthorised premises, establishment and areas of worshipagglomerations, among others

- Identification of groups and communities that are vulnerable or especially sensitive to the pandemic

- Identification of shortages and supply of basic resources

- Prediction of areas of potential contagion for reasons of large influx, presence of diagnosed cases, and lack of socio-health measures

- Creation and provision of a real-time report of observations and suspicions of COVID-19 by the population

- Facilitate access to social network data on the epidemic for the scientific community to support artificial intelligence, simulation and epidemiology.

The possible digital data of an individuals can be collected from the sensors of his smart's phones, wearables, social medial platforms through the open artificial intelligence (AI). The system shall aggregates the data and push it into the analytical format to the analytics unit where various analytics to be performed to get various insights and those insights should be update to the monitoring or controlling authorities and finally publish it into the social sites along with the real-time alarms to the respective users. The location of the study is Bangalore, Karnataka, India. The anticipated outcome of study is a unified design architecture for the realization of the application which can be implemented first as a prototype and later in real time scenario. Therefore, the scope of this research is to arrive a well-documented realizable architecture of an application to enforce social distance in real-time scenario.The proposed study also offers a relevance to future strategies. The architecture will a well-crafted blue print of a crowd-cum social data sourcing based social distancing mechanism which can be customized for small society to the town to the nationwide movements of economical movements along with social distancing so that physical health and the economic health of the nation is maintained during and post pandemic era. The architectural diagram of the methodology adopted for the proposed system is shown in the Figure 1.

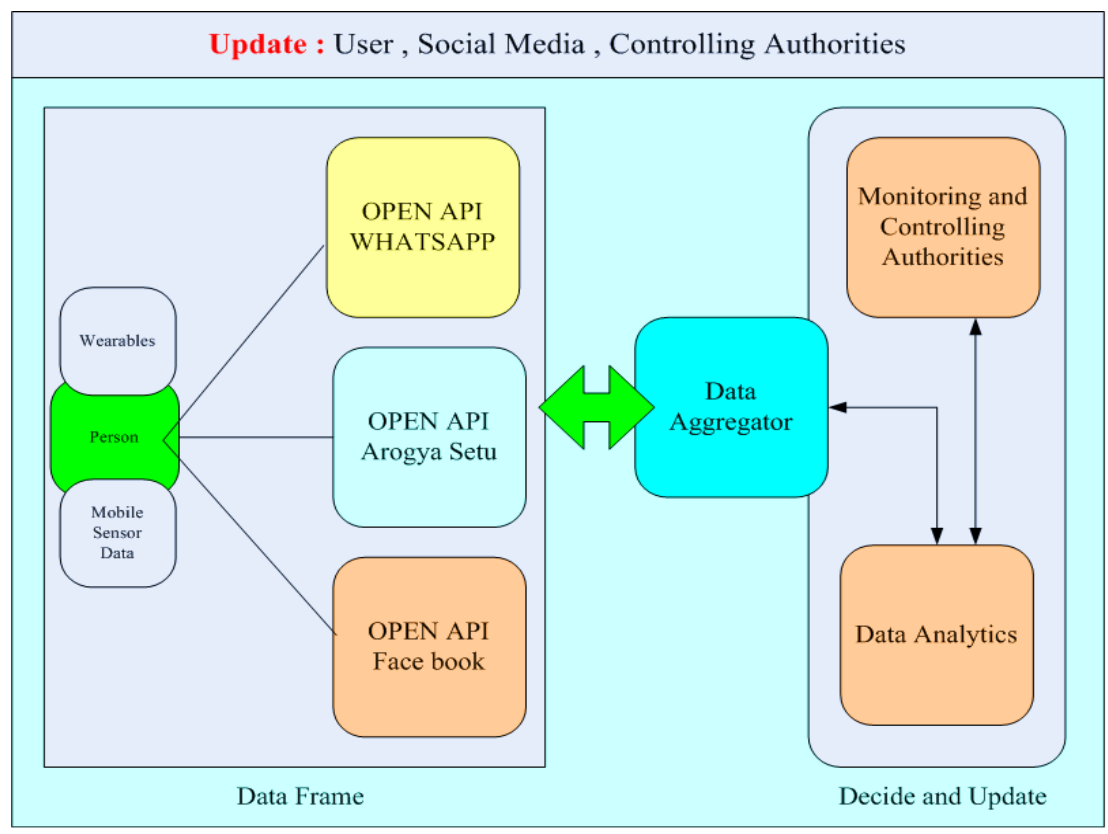

Figure 1. Architectural diagram of the tentative methodology for the proposed system 


\section{PROPOSED METHOD}

The system design of proposed study is shown in Figure 2 which consists of essential actors of the study i.e. i) a handheld device for user, ii) cloud services with storage servers, and iii) medical authorities. The proposed system could use a simplified android app (Arogya Setu) where the user enrolls their details. A Bluetooth is used for this purpose to represent its presence where the environment of broadcasting Bluetooth signal will consist of an arbitrary identity with respect to a trusted handheld device where the enrollment details are hosted over the cloud services. The system resists unwanted monitoring of the handheld device by altering this arbitrary identity after specific duration of time. All the incoming signals from the Bluetooth in proximity are sensed by the registered handheld device of user which is consistently in listening mode. In this process, the system senses the signal strength of the Bluetooth signal and all the instantaneous time with respect to the contact with other users. Apart from this, the GPS location of the user is also tracked which is only enabled by the permission set mutually by the user. The system facilitates a preliminary assessment of the threat upon counter interacting with others user.

An active communication channel is used for forwarding the aggregated data autonomously by the handheld device to the cloud server in order to next level of threat assessment. A real-time procedure is used for carrying out this data transmission where various wireless communication systems including internet connection can be used for the transmission of the sensed data. Any confirmed case of COVID-19 by the national database system is further updated by national medical authorities. The accurate outcome is obtained by using particular algorithm which is hosted in the cloud server. All the contact and medical history of the user can be then accessed by the medical officer up to specific period of time where the data is retained associated with the vulnerable user. This process allows the cloud service to compute the level of vulnerability using predictive algorithm of all the direct contacts of the vulnerable user along with their current location, which is not present in existing Arogya Setu app. All the contacts are then notified by the proposed system as per the level of threat and offer an elaborated guidance to countermeasure the existing threat. One of the interesting contributions of the proposed system is the exhibits of all the contact as well as all vulnerable users to upscale or downscale their social distancing.

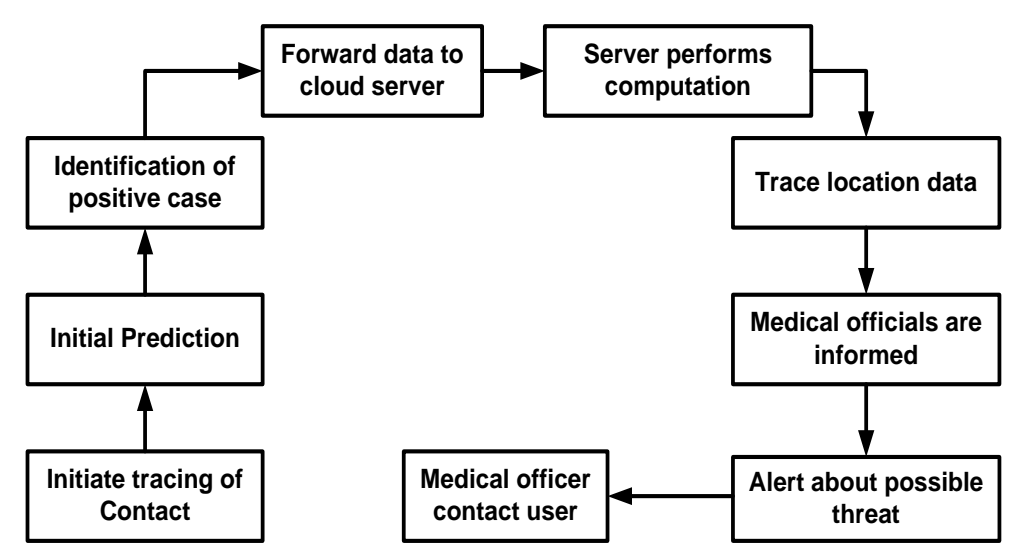

Figure 2. Proposed system design

\subsection{Formulating social distancing}

The proposed system emphasized on the spatial distance between two contacts as the prime parameter to formulate a new measure for the social distancing. The proposed system makes use of the received signal strength indicator in order to compute the effective social distance between any signals which comes in the range of the Bluetooth. Hence, the proposed system make used of signal power and spatial distance in order to formulate social distancing which is mathematically expressed as (1).

$$
\tau_{\mathrm{rec}}=\tau_{1}-\mu
$$

In the mathematical expression (1), the first variable $\tau_{1}$ will represents received signal power empirically represented by decibel-meter. The second variable $\mu$ will represent a product of loss parameter and logarithmic function of spatial distance between two contacts within the proximity of Bluetooth signal. The study considers 1 meter interval as the received signal strength indicator and distance of 15 meters is considered in the form of spatial range. 


\subsection{Prediction of threat probability}

The proposed study constructs a simplified mechanism in order to compute the probability of threat (higher degree of infection) with respect to specific user. The proposed system carry out this predictive modelling on the basis of contact period between the two signals along with the spatial distance between two users using probability concept. The proposed study empirically assumes (2):

$$
\operatorname{Prob}_{\text {dist }}(d)=e^{-c 1 d}
$$

In the mathematical expression (2), the variable $c l$ and $d$ represents positive network coefficient and distance. The above expression is formed for assessing the various degree of fluctuation of all the possibilities of getting in communication radius of user with reported threat of positive COVID-19 case. The above mentioned distribution of the predictive data is characterized by the minimization of all possibility of getting infected with maximization of the two contacts. It will mean that this formulation will yield a necessary social distancing required to be maintained by the normal user when they come in contact with the COVID-19 positive case. Hence, the proposed system computes probability of variation in the identification process considering contact period and it is mathematically expressed as in (3).

$$
\operatorname{Prob}_{\mathrm{dur}}(\text { per })=\Delta \mathrm{e}^{-\mathrm{c} 2 \mathrm{per}}
$$

In the mathematical expression (3), the variable $c 2$ and $d u r$ represents the positive network coefficient and period when two user comes in contact with each other. The computation of this probability attribute will yield distribution of the probability characterized by maximized feasibility of getting closer to infected user with more period of contact. Figure 3 highlights the pictorial presentation of tracing the contact.

Figure 3 highlights the contact between user-a and user-b with the presence of user-a in r-tier and user- $b$ located in $(\mathrm{r}+1)$ tier. The picture highlights dis $\mathrm{s}_{\mathrm{ab}}$ as the distance of contact between two users as well as per $_{\text {ab }}$ period between two users in contact. The proposed system compute the probability that the user-b can get infected from user-a by obtaining a product of these probabilities indicated by Prob(user-b, user-a) along with the cumulative probability of obtaining an exposure to the infected user Prob. The mathematical expression for this probability is as (4).

$$
\operatorname{Prob}\left(\text { user-b, user-a)=Prob(user-a) } \text { prob }_{\text {dist }}\left(\text { dist }_{\mathrm{a}, \mathrm{b}}\right) \cdot \operatorname{prob}_{\text {per }}\left(\text { per }_{\mathrm{a}, \mathrm{b}}\right)\right.
$$

The mathematical expression (4) computes the probability of getting one user when they come under a specific range and stay within that range of the infected user. However, the proposed method considers medical condition of the user apart from period and spatial distance if the user chooses to share this additional information with the application. The proposed study consider this as $\rho$ which contributes towards probability assessment represented by Prob $_{\rho}$, which is mathematically represented as in (5).

$$
\operatorname{Prob}(\text { user-b, user-a })=\arg _{\min }\left(\operatorname{Prob}\left(\mathrm{user}_{-\mathrm{a}}\right) \cdot \operatorname{Prob}_{\mathrm{dist}}\left(\operatorname{dist}_{\mathrm{a}, \mathrm{b}}\right) \cdot \operatorname{Prob}_{\mathrm{dur}}\left(\operatorname{dur}_{\mathrm{a}, \mathrm{b}}\right)+\Sigma \operatorname{Prob}_{\rho}\right)
$$

In the mathematical expression (5), the study considers presence of specific number of additional contributory medical information. This additional information can be obtained from social network application as well as Whatsapp messaging service. The proposed system constructs a framework which uses Bluetooth signal as well as location information of the user. The idea is to track the complete information about the distance and period of contact. The proposed system develops a computational framework which is initialized with enrollment of user where email-id, address, mobile number of the user is stored. The user will be prompted to add additional medical information which is optional for the user in order to carry out an effective prediction of the status of threat for the user for COVID-19. Once the enrollment of the first time user is over, the framework is required to be running on the background of all application in user handheld device. The proposed framework also ensure that private information of the user to be maintain as a secret by the user by configuring the privacy setting especially the location information of the user. This framework assists the Bluetooth signaling mode along with monitoring mode while they operate simultaneously. The signaling values of the received signal strength associated with the Bluetooth in proximity is assessed along with the location information from the GPS with timestamped data captured on ease scanning. This information is stored in the handheld device of the user. Apart from this, cumulative duration of the contact for the entire handheld device in the proximity is also read. This forms the aggregated data using various technologies supported by the handheld device of the user. After the availability of the network connectivity with sufficient channel capacity, this information is forwarded to the cloud server. The next part of the implementation is to subject the aggregated data to the data analytics module which make use of deep neural 
network which is currently a dominant technology in machine learning. The prime motive of using deep neural network is mainly to confirm the accuracy of the threat computed by the probability in proposed study. The proposed system can easily assume that there will be massive generation of the identified threat from the proposed threat assessment and identification system. Hence, it is further required to offer more precision to confirm if the threat is actually lethal or is an outlier. Apart from this, it is also necessary to perform classification of the form of the threat.

In the proposed system of data analytics as shown in Figure 4, after the incoming aggregated data is fed to the neural processor, the transformation operation is applied for all the levels of aggregated data in order to obtain an abstractive data with highly compact representation. In the process of threat identification and classification of COVID-19, the raw input of the aggregated data is formulated in the form of matrix where the initial layer of representation performs threat abstraction followed by encoding the information. The second layer further performs composition followed by encoding of the edges of the network. The third layer performs encoding of all the primary contact information along with duration of contact, while the fourth layer perform identification of actual threat. In this case, the processor is equipped with national medical database to represent the actual medical representation of level of threats. Prior to giving a predictive outcome by the deep neural network model, the outcome of the processor is cross-checked with the standards of the medical condition to finally offering the classified outcome of higher threat, moderate threat, and low threat score. The proposed study makes use of probability theory in order to express the final outcome and assess their threat significance. The next section discusses about the outcome obtained from proposed system.

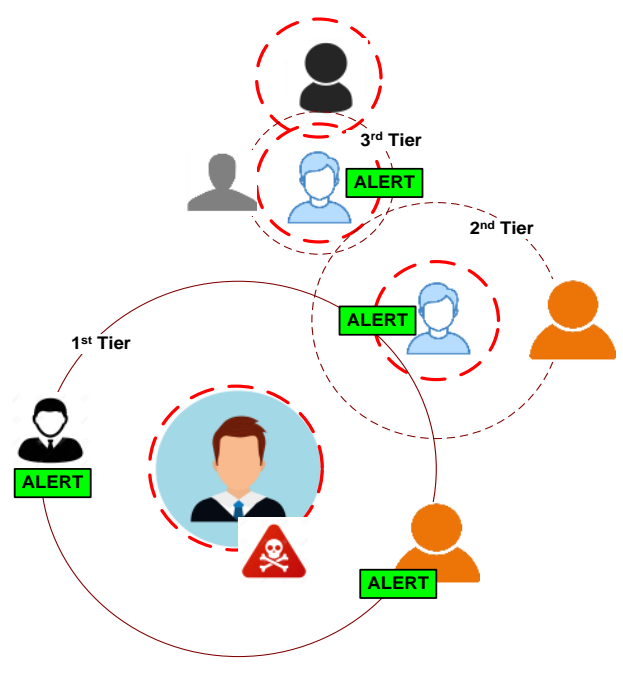

Figure 3. Mechanism of tracing contact

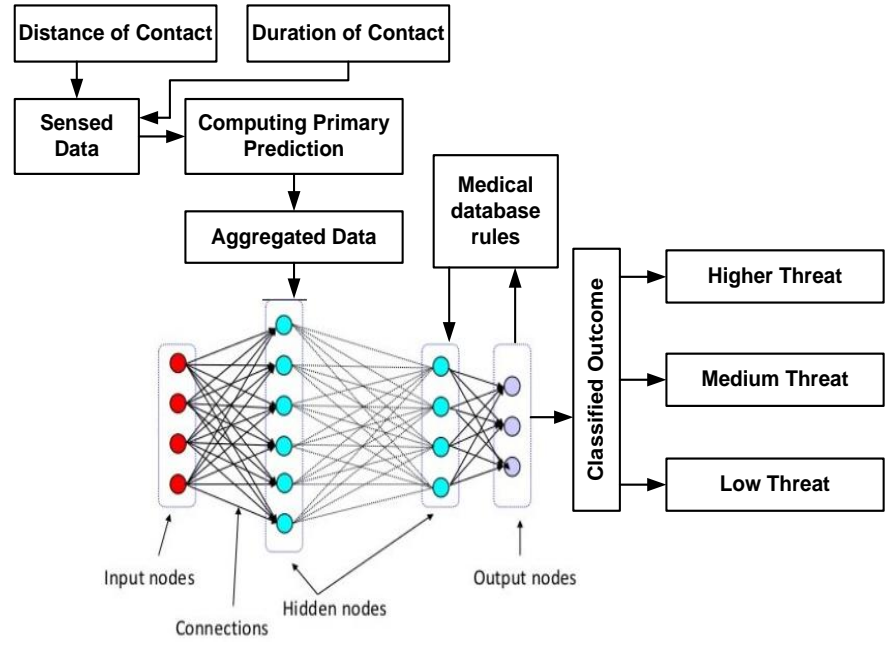

Figure 4. Proposed data analytical operation

\section{RESULTS AND DISCUSSION}

This section discusses about the outcome obtained from the proposed study. A framework is developed in MATLAB where the received signal strength is considered to be at interval of 1 meter and spatial distance of 10 meters. The readings of the received signal strength indicator are considered for 50 times for all the position within a range. A prototyping of mobile application is designed using android development toolkit, which runs the mathematical expression discussed in prior section. All the data are extracted and forwarded to cloud server which host the data analytical module using deep neural network. The study outcome is validated by comparing with three conventional predictive approaches i.e. artificial neural network (ANN), support vector machine (SVM), and k-nearest neighborhood (KNN). The assessment is carried out using 4 appropriate performance parameters i.e. energy consumed by the handheld device, usage of RAM, accuracy of classification, and processing time of complete approach as shown in Figure 5.

Figure 5(a) highlights that proposed system offer less energy consumption for the user handheld device as compared to other predictive approach. This is because of faster learning and classification operation carried out by deep neural network in proposed system, whereas the process of learning and error minimization is too much iterative in existing approach. Similar justification is also for the outcome of Figure 5(d). Figure 5(b) highlights about memory performance. It should be noted that the proposed study has been assessed on 64 GB internal memory handheld device. The memory dependency only till the aggregated data is not forwarded to cloud server. The application does not store any intermediate information 
in its memory and hence it is quire lightweight applicable for any form of handheld device. Figure 5(c) highlights about the accuracy performance of proposed system. Owing to its independence from extracting significant feature from the aggregated data, deep neural network is found to be ideal for this condition as it does not need any form of feature to perform classification. Rather, it is capable of extracting its own feature to offer significantly higher accuracy performance. However, learning time is quite high for SVM which leads to increased consumption of processing time in Figure 5(d). Apart from this, ANN has always an uncertainty towards the learning process while KNN has the dependency of quality data to offer increasing accuracy. It also has higher memory dependencies. Therefore, on the basis of the mentioned results, it can be said that the proposed system offers not only a cost effective predictive operation but also highly accurate predictive outcomes. Hence, from this perspective, proposed system offers better alternative compared to using the existing mobile application of social distancing.

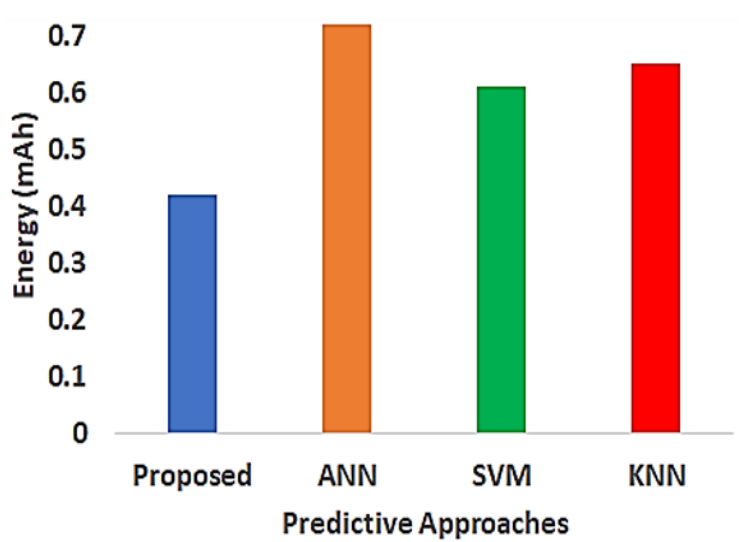

(a)

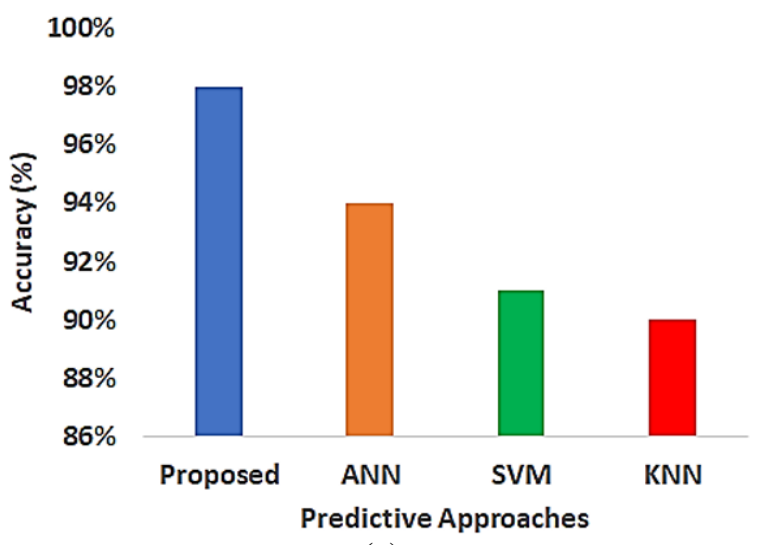

(c)

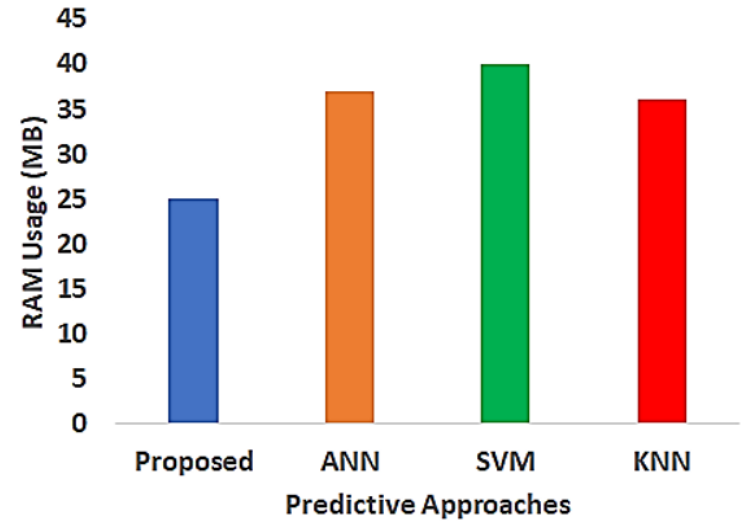

(b)

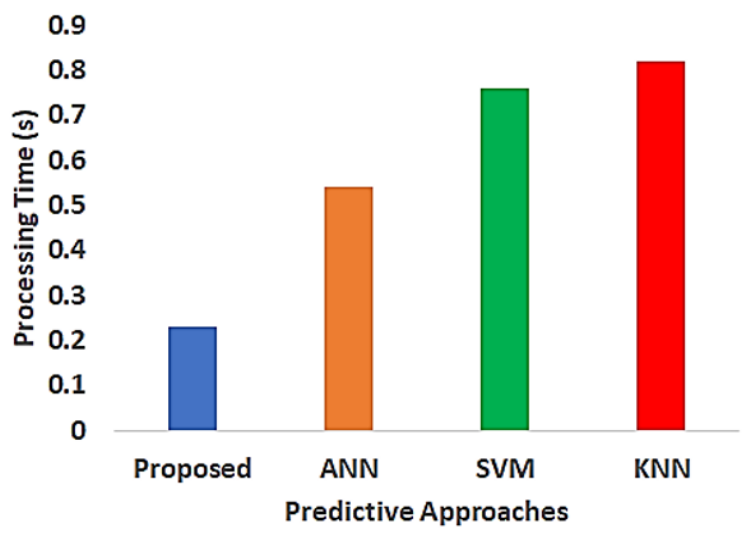

(d)

Figure 5. Analysis of comparative analysis: (a) energy performance, (b) RAM usage, (c) classification accuracy, (d) processing time

\section{CONCLUSION}

This paper has discussed about the simple and cost-effective mechanism of modernizing the concept of social distancing slightly different and innovative manner from existing applications. The contribution of the study are viz: i) unlike existing Arogya Setu mobile application, this application considers duration of contact apart from the proximity distance, ii) the proposed model considers GPS based tracking system in order to find the current location of infected person which cannot be found in Arogya Setu mobile application, iii) different from Arogya Setu, the framework considers information from social networking application for facilitating better precision, iv) the proposed model is capable of applying deep learning towards the aggregated data in order to offer better classification of level of threats, and v) based on the classified outcome, customized social distancing norms for individual is sent as an alert whereas in existing system Arogya Setu just make of same template of alert for everyone. 


\section{REFERENCES}

[1] K. Mukund, K. Mathee, and S. Subramaniam, "Plasmin cascade mediates thrombotic events in SARS-CoV-2 infection via complement and platelet-activating systems," IEEE Open Journal of Engineering in Medicine and Biology, vol. 1, pp. 220-227, 2020, doi: 10.1109/OJEMB.2020.3014798.

[2] F. Alizadeh and A. Khodavandi, "Systematic review and meta-analysis of the efficacy of nanoscale materials against coronaviruses-possible potential antiviral agents for SARS-CoV-2," IEEE Transactions on NanoBioscience, vol. 19, no. 3, pp. 485-497, 2020, doi: 10.1109/TNB.2020.2997257.

[3] A. J. Jinia et al., "Review of sterilization techniques for medical and personal protective equipment contaminated with SARSCoV-2," IEEE Access, vol. 8, pp. 111347-111354, 2020, doi: 10.1109/ACCESS.2020.3002886.

[4] G. Pinkas, Y. Karny, A. Malachi, G. Barkai, G. Bachar and V. Aharonson, "SARS-CoV-2 detection from voice," IEEE Open Journal of Engineering in Medicine and Biology, vol. 1, pp. 268-274, 2020, doi: 10.1109/OJEMB.2020.3026468.

[5] S. Meerdith. "Harvard researchers warn social-distancing measures may need to remain in place into 2022." CNBC.com. 2020. https://www.cnbc.com/2020/04/15/coronavirus-study-warns-social-distancing-may-need-to-stay-until-2022.html (Accessed Mar 5, 2021).

[6] Statista Research Department. "COVID-19 cases in india as of july 2021." Statista.com. 2021. https://www.statista.com/statistics/1101713/india-covid-19-cases-by-type (accessed May, 2021).

[7] L. Rafea, A. Ahmed, and W. D. Abdullah, "Classification of a COVID-19 dataset by using labels created from clustering algorithms," Indonesian Journal of Electrical Engineering and Computer Science (IJEECS), vol. 21, no. 1, pp. 164-173, 2021, doi: 10.11591/ijeecs.v21.i1.pp164-173.

[8] M. Odeh, and M. Yousef, "The effect of Covid-19 on the electronic payment system: usage level trust and competence perspectives," Indonesian Journal of Electrical Engineering and Computer Science (IJEECS), vol. 22, no. 2, pp. 1144-1155, 2021, doi: 10.11591/ijeecs.v22.i2.pp1144-1155.

[9] A. A. Ojugo and R. E. Yoro, "Extending the three-tier constructivist learning model for alternative delivery: ahead the COVID-19 pandemic in Nigeria," Indonesian Journal of Electrical Engineering and Computer Science (IJEECS), vol. 21, no. 3, pp. 1673-1682, 2021, doi: 10.11591/ijeecs.v21.i3.pp1673-1682.

[10] M. Adebiyi and O. O. Olugbara, "Binding site identification of COVID-19 main protease 3D structure by homology modeling," Indonesian Journal of Electrical Engineering and Computer Science (IJEECS), vol. 21, no. 3, pp. 1713-1721, 2021, doi: 10.11591/ijeecs.v21.i3.pp1713-1721.

[11] D. M. Mason, M. Kapinaj, A. P. Martínez, and L. Stella, "Impact of social distancing to mitigate the spread of COVID-19 in a virtual environment," ACM Symposium on Virtual Reality Software and Technology, 2020, pp. 1-3, Art. no. 59, doi: $10.1145 / 3385956.3422093$.

[12] T. Oransirikul and H. Takada, "Social distancing warning system at public transportation by analyzing wi-fi signal from mobile devices," Adjunct Proceedings of the 2020 ACM International Joint Conference on Pervasive and Ubiquitous Computing and Proceedings of the 2020 ACM International Symposium on Wearable Computers, 2020, pp. 267-271, doi: $10.1145 / 3410530.3414361$.

[13] M. Wiberg, "On physical and social distancing: reflections on moving just about everything online amid Covid-19," Interaction, vol. 27, no. 4, pp. 38-41, 2020, doi: 10.1145/3404213.

[14] R. J. Milne, C. Delcea, L. -A. Cotfas, and C. Ioanăş, "Evaluation of boarding methods adapted for social distancing when using apron buses," IEEE Access, vol. 8, pp. 151650-151667, 2020, doi: 10.1109/ACCESS.2020.3015736.

[15] C. T. Nguyen et al., "A comprehensive survey of enabling and emerging technologies for social distancing-part i: fundamentals and enabling technologies," IEEE Access, vol. 8, pp. 153479-153507, 2020, doi: 10.1109/ACCESS.2020.3018140.

[16] M. Cristani, A. D. Bue, V. Murino, F. Setti, and A. Vinciarelli, "The visual social distancing problem," IEEE Access, vol. 8, pp. 126876-126886, 2020, doi: 10.1109/ACCESS.2020.3008370.

[17] P. B. Brandtzaeg, "How mobile media impacts urban life: Blending social cohesion and social distancing," Interaction, vol. 27, no. 6, pp. 52-56, 2020, doi: 10.1145/3424682.

[18] B. Pejó and G. BiczóK, "Corona games: Masks, social distancing and mechanism design," Proceedings of the 1st ACM SIGSPATIAL International Workshop on Modeling and Understanding the Spread of COVID-19, 2020, pp. 24-31, doi: $10.1145 / 3423459.3430757$.

[19] N. S. Punn, S. K. Sonbhadra, and S. Agarwal, "Monitoring COVID-19 social distancing withperson detection and tracking via fine-tuned YOLOv3 and Deepsort techniques," arXiv:2005.01385, 2020.

[20] M. Rezaei and M. Azarmi, "DeepSOCIAL: Social distancing monitoring andinfection risk assessment in COVID-19 pandemic," Applied Sciences, vol. 10, no. 21, 2020, Art. no. 7514, doi: 10.1101/2020.08.27.20183277.

[21] V. Chamola, V. Hassija, V. Gupta, and M. Guizani, "A comprehensive review of the COVID-19 pandemic and the role of IoT, drones, aI, blockchain, and 5G in managing its impact," IEEE Access, vol. 8, pp. 90225-90265, 2020, doi: 10.1109/ACCESS.2020.2992341.

[22] A. Ghimire, S. Thapa, A. K. Jha, A. Kumar, A. Kumar, and S. Adhikari, "AI and IoT solutions for tackling COVID-19 pandemic," 2020 4th International Conference on Electronics, Communication and Aerospace Technology (ICECA), Coimbatore, India, 2020, pp. 1083-1092, doi: 10.1109/ICECA49313.2020.9297454.

[23] K. Hussain, X. Wang, Z. Omar, M. Elnour, and Y. Ming, "Robotics and artificial intelligence applications in manage and control of COVID-19 pandemic," 2021 International Conference on Computer, Control and Robotics (ICCCR), Shanghai, China, 2021, pp. 66-69, doi: 10.1109/ICCCR49711.2021.9349386.

[24] Q. Pham, D. C. Nguyen, T. Huynh-The, W. Hwang, and P. N. Pathirana, "Artificial intelligence (AI) and big data for coronavirus (COVID-19) pandemic: A survey on the state-of-the-arts," IEEE Access, vol. 8, pp. 130820-130839, 2020, doi: 10.1109/ACCESS.2020.3009328.

[25] V. Chamola, V. Hassija, S. Gupta, A. Goyal, M. Guizani, and B. Sikdar, "Disaster and pandemic management using machine learning: A survey," IEEE Internet of Things Journal, pp. 1-1, 2020, doi: 10.1109/JIOT.2020.3044966.

[26] V. R. J and A. Jakka, "Forecasting COVID-19 cases in India using machine learning models," 2020 International Conference on Smart Technologies in Computing, Electrical and Electronics (ICSTCEE), Bengaluru, India, 2020, pp. 466-471, doi: 10.1109/ICSTCEE49637.2020.9276852.

[27] A. K. Bairagi et al., "Controlling the outbreak of COVID-19: A noncooperative game perspective," IEEE Access, vol. 8, pp. 215570-215581, 2020, doi: 10.1109/ACCESS.2020.3040821.

[28] D. McAdams, K. K. McDade, O. Ogbuoji, M. Johnson, S. Dixit, and G. Yamey, "Incentivising wealthy nations to participate in the COVID-19 vaccine global access facility (COVAX): A game theory perspective," BMJ global health, vol. 5, no. 11, 2020, doi: 10.1136/bmjgh-2020-003627. 
[29] A. Narin, "Detection of covid-19 patients with convolutional neural network based features on multi-class x-ray chest images," 2020 Medical Technologies Congress (TIPTEKNO), Antalya, Turkey, 2020, pp. 1-4, doi: 10.1109/TIPTEKNO50054.2020.9299289.

[30] M. Mousavi, R. Salgotra, D. Holloway, and A. H. Gandomi, "COVID-19 time series forecast using transmission rate and meteorological parameters as features," IEEE Computational Intelligence Magazine, vol. 15, no. 4, pp. 34-50, Nov. 2020, doi: 10.1109/MCI.2020.3019895.

\section{BIOGRAPHIES OF AUTHORS}

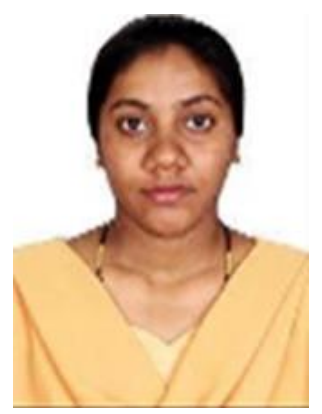

Salma Firdose (iD 8D SC P Working as an Assistant Professor in Department of Computer Science at Christ Academy Institute of Advanced Studies, Bangalore (Sep. 2020 - till date). She has completed her Doctor of Philosophy (Ph.D.) in Software Engineering from Bharathiar University, 2019. She did her Bachelor of Science from Bangalore University from 2000 to 2003.In 2003 received the Bachelor degree. She Studied Masters of Computer Application from Bangalore University from 2003 to 2006 and was awarded masters in the same year.in 2007 to 2009 did Master of Philosophy from Bharathiar Universiy, Coimbatore. She can be contacted at email: salmaf@ caias.in.

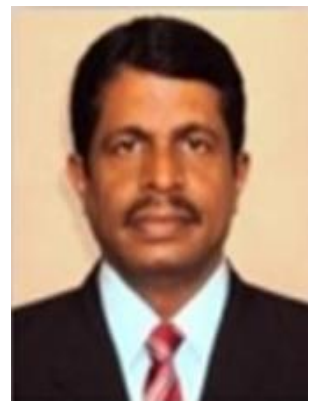

Surendran Swapna Kumar (D) SC P completed his Ph.D. in the department of Department of Electronics and Communication Engineering from Anna University. He has published more than 25 national and international papers. He got some awards Selected for Best Paper Category (GOLD) of the "Second International Conference on Advances in Recent Technologies in Communication and Computing" organized by the Association of Computer Electronics and Electrical Engineers (ACEEE) - a division of Engineers the October 2010 and presented paper on "Implementation of Hybrid Ad-Hoc Routing Protocol”. He can be contacted at email: swapnakumar.s@vidyaacademy.ac.in.

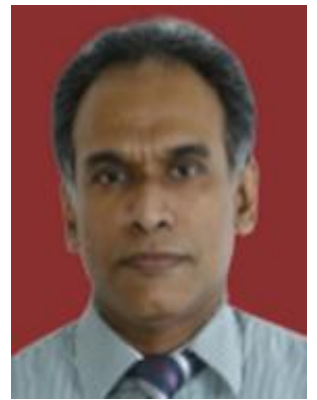

Ravinda Gayan Narendra Meegama (D) SOS SC $\mathrm{P}$ working as a professor in Computer Science Department of Computer Science Faculty of Applied Sciences, University of Sri Jayewardenepura Gangodawila, Nugegoda, Sri Lanka. He has completed his Ph.D. in computer science in the year of 2004 from Nanyang Technological University, Singapore. $\mathrm{He}$ is having $20+$ years teaching experience in the university. He has published 76 national and international papers. His area of speciazations is in Software Engineering, Networking, and Computer Graphics. He can be contacted at email: rgn@sci.sjp.ac.lk. 